RESEARCH ARTICLE

\title{
Isokinetic Force and Work Capacity after Long-Duration Station "MIR» and Short-Term International Space Station Missions
}

\author{
Yuri A Koryak* \\ Department of Sensory-motor Physiology and Countermeasures, Institute of Biomedical Problems of the \\ Russian Academy of Sciences, Russia
}

*Corresponding author: Koryak Yu, State Scientific Center (SSC) of the Russian Federation, Institute of Biomedical Problems of the Russian Academy of Sciences (RAS), Department of Sensory-motor Physiology and Countermeasures, Laboratory of Gravitational Physiology of Sensory-motor system, Khoroshevskoye Shosse 76-A, 123007 Moscow, Russia

\begin{abstract}
Introduction: The effects of long-duration ( 210 days) aboard the Orbital Station "MIR" and short-term ( 10 days) spaceflights aboard the International Space Station on the joint torques of various muscles and work capacity of knee extensors were studied in male cosmonauts.

Methods: Joint torque and muscle endurance testing was performed 30 days before and 3-5 days after a spaceflight, using a LIDO ${ }^{\circledR}$ Multi-Joint Isokinetic Rehabilitation System (USA).

Results: Greater postflight changes in maximal joint torque were observed for back, knee, and ankle extensors compared with flexors, and the difference was especially clearly seen after long-term spaceflights. Muscle work capacity was inferred from the gradient of declining muscle force produced in a series of rhythmic voluntary concentric movements and was found to decrease after both shortand long-term spaceflights. The area under the muscle contraction curve decreased to a greater extent and in all regions of the curve after long-term spaceflights.

Discussion: The finding that the contractile functions and work capacity of muscles decrease more after long-term than after short-term spaceflights in spite of the physical training program of a certain type gave grounds to assume that physical training employed in long-term spaceflights were insufficient to simulate the daily mechanical load that the cosmonauts had before a spaceflight.
\end{abstract}

\section{Keywords}

Real microgravity, Spaceflight, Contractile properties, Isokinetic dynamometry

\section{Introduction}

A microgravity due to actual (spaceflight) or simulated microgravity (bed rest, immersion, or unilateral lower limb unloading) provides a unique environment where forces of body interactions with a support are absent, causing physiologically adverse problems to various body systems and, in particular, the locomotor system. Extremely complex changes arise in muscle mass, and contractile function in microgravity in spite of Physical Training (PT) employed during longduration spaceflights, as has been demonstrated in many studies. The loss of skeletal muscle mass [1] and concomitant changes in muscle function [2] induced by prolonged sojourns in microgravity environments can adversely affect the performance of astronauts during routine mission-related activities and upon their return to Earth.

Muscle mass loss of $4-10 \%$ has been observed in astronauts after an 8-day Shuttle mission [3]. The muscle volume has been reported to decrease to a somewhat greater extent, by $6-16 \%$, with the spaceflight duration increasing from 9 to 16 days [4]. Moreover, muscle fiber atrophy of $16-36 \%$ has been found in $m$. vastus lateralis after an 11-day spaceflight [5].

Greater losses of muscle mass occur during longer spaceflights in spite of employing PT programs. For instance, a decrease of $10-24 \%$ in muscle volume has been observed in astronauts after missions of approximately 6 month to the "MIR" space station and 
the International Space Station (ISS) [1,6-8]. However, the muscle mass has been found to stabilize to a certain extent or to achieve a new stable level in humans exposed to 120-day antiorthostatic bed rest [1], which is used as a main ground-based model of longduration spaceflights to study the long-term effects of microgravity [9].

The strength of muscle contraction decreases in parallel with muscle volume loss, but the changes in strength are somewhat greater [10-15]. For instance, a substantial (up to $14 \%$ ) decrease in hand muscle contraction strength has been observed after short-term (2-5 days) spaceflights aboard Soyuz spacecrafts [16], and decreases of $12 \%$ in the strength of hip extensors, $23 \%$ in the strength of hip flexors $[17,18]$, and up to $16 \%$ in muscle work capacity $[1,3,4,7,19]$ have been reported for astronauts after Shuttle missions of 5-17 days.

Studies of the force-velocity characteristics of ankle extensors and flexors by conventional iso kinetic dynamometry after a 7-day spaceflight have shown a decrease in the strength of ankle extensors throughout the velocity range examined, including an isokinetic regimen, the decrease amounting to $20-30 \%$ of the baseline in all but the isometric mode and being somewhat lower, $\sim 15 \%$, in the isometric regimen [2022].

Postflight measurements in crew members after missions of 16-28 weeks to the "MIR" space station have shown a decrease of up to $48 \%$ in the force of Maximal Voluntary Contraction (MVC) of ankle flexors [2] an a $31 \%$ increase in the maximal shortening velocity of muscle fibers [23]. The contraction force of knee extensors and the endurance of knee flexors have been found to decrease by approximately $26 \%$ after a mission of 129-145 days to the ISS [24]. Trappe, et al. [25] have reported decreases in maximal power (by $32 \%$ ) and maximal force (by 20-29\%) of ankle extensors in voluntary movements in a broad isokinetic velocity range after a mission of approximately 6 month to the ISS. It is commonly accepted that major losses affect trunk and lower limb muscles, which are highly active under normal Earth's gravity (1 G). Muscle strength in the upper limbs is affected to a lesser extent than that in the lower limbs $[20,26]$.

The fact that losses in muscle strength are greater than losses in muscle mass (volume) after both spaceflight $s$ and model exposures (immersion, bed rest, or limb suspension) has prompted a hypothesis that the motor control is basically affected in microgravity [2729]. For instance, a $45 \%$ decrease in power produced by hip extensors after a 180-day spaceflight is considerably greater than can be explained solely by muscle mass loss [29]. A decrease in power produced by hip extensors during explosive voluntary contractions is accompanied by a substantial decrease in their Electromyographic (EMG) activity [29]. Zange, et al. [8] have observed a greater percent change in force (by 20-48\%) than in muscle volume (by 6-20\%) for knee extensor by magnetic resonance imaging. Lambertz, et al. [23] have similarly reported a $17 \%$ decrease in isometric force after $90-180$ days.

A decrease in muscle contraction force has been accompanied by a decrease in EMG activity of ankle extensors, averaging 39\% [23]. Concomitant changes observed after spaceflights have similarly been detected in ground-based model studies simulating muscle unloading. For instance, Hather, et al. [30] have shown using MRI that a 6-week unilateral unloading of the left leg reduced the cross-sectional area (CSA) of its hip extensors by $12 \%$ relative to the right leg, which was not unloaded. The total muscle CSA of the left leg was $14 \%$ lower than that of the right leg. MRI showed irregular reduction of the CSA for thigh muscles. The CSA reduction in hip extensors $(-16 \%)$ was twice greater than in hip flexors $(-7 \%)$. Interestingly, $m$. rectus femoris did not display any CSA reduction, while all three heads of $m$. vastus femoris showed a reduction of approximately $16 \%$. The total CSA reduction in the unloaded limb was assumed to be due mostly to the CSA reduction in $\mathrm{m}$. soleus $(-17 \%)$ and $\mathrm{m}$. gastrocnemius (-26\%). Berg, et al. [12] have observed that the isometric MVC and concentric forces of hip extensors were reduced by $25-30 \%$ after bed rest for 42 days, while the CSA decreased only by $14 \%$; and maximal EMG activity, by $19 \%$.

Greater changes in muscle strength than in CSA suggest changes in internal properties [31] and of motor units, their recruitment, or neural drive [11,3234]. The last assumption is supported by a lower electromechanical efficiency, which is evident from the finding that higher EMG activity (+ 44\%) is involved in generating the same isometric force $(100 \mathrm{Nm})$ after 6 -week bed rest $[12,35]$. Therefore, changes in muscle activation may substantially affect the operational ability of crew members upon landing after exposure to microgravity.

Here were report the changes observed in the maximal joint torques of several muscle groups and the muscle work capacity of nine crew members after MIR missions $213.0 \pm 30.5$ days and five visiting crew members after ISS missions of 7-10 days.

A unique feature of this study is that changes in contractile properties are directly compared for spaceflights of different durations and muscular alterations are considered in the context of using or not using PT during a spaceflight.

The objective of the study was to quantitatively estimate the changes in contractile functions and work capacity of muscles in cosmonauts after shortterm and long-duration spaceflights by using isokinetic dynamometry during voluntary contractions. In the 
current study, we report changes in the strength skeletal muscle strength, and endurance (performance) in nine crewmembers missions of 213 days to the MIR and in five crewmembers missions of 10 days to the ISS. We assumed that the large changes will be observed after a long-duration spaceflights and a greater degree in of extensors muscles.

\section{Methods}

\section{Familiarization}

The cosmonauts reported to the laboratory twice. Each subject received verbal explanations of the study protocols. The cosmonauts gave their written informed consent to participate in this study. During the first visit, the cosmonauts were familiarized with the experimental set-up and procedures during a preliminary session before starting the preflight tests [ 60 day preflight (L60)]. On a subsequent visit the cosmonauts performed two sets of experiments $\sim 40-30$ days before spaceflight [Baseline Data Collections (BDC)] and immediately after spaceflight [return (+ R); +R5/+ R7].

All experimental procedures complied with the Declaration of Helsinki (1975). The study protocol was approved by the Committee for the Protection of Human Subjects of NASA's Johnson Space Center, Houston, TX, and by the Biomedical Ethics Committee at of the State Scientific Center - Institute of Biomedical Problems, RAS, Moscow, and by Yu.A. Gagarin Cosmonauts Training Center, Star City, Moscow Region, Russia.

\section{Subjects}

Two groups of male cosmonauts participated in the study. Group $1(n=9 ; 45.1 \pm 2.0$ years, $176.0 \pm 2.3 \mathrm{~cm}$, $79.9 \pm 2.0 \mathrm{~kg}$ ) included prime crew members from longduration missions ( $209.8 \pm 30.4$ days) to the "MIR" space station; group 2 ( $n=5 ; 37.6 \pm 2.8$ years, $177.0 \pm 1.8 \mathrm{~cm}$, $74.02 .8 \mathrm{~kg}$ ) included visiting crew members from shortterm missions ( $9.4 \pm 0.2$ days) to the ISS (Table 1 ). All crew members were informed about the goals and methods of the study of muscle contractile functions, the study procedures, risks, and study significance, and gave their written informed consent to voluntary participation in the study as subjects.

The cosmonauts did not do PT during short-term missions and followed a standard Russian PT program during long-duration spaceflights [36,37].

Table 1: Physical and anthropometric characteristics of the members space missions.

\begin{tabular}{|l|l|l|l|l|}
\hline № & $\begin{array}{l}\text { Age, } \\
\text { year }\end{array}$ & $\begin{array}{l}\text { Height, } \\
\text { cm }\end{array}$ & $\begin{array}{l}\text { Body mass, } \\
\text { kg }\end{array}$ & $\begin{array}{l}\text { Space flight, } \\
\text { day }\end{array}$ \\
\hline First group & & & & \\
\hline M & $\mathbf{4 5 . 1}$ & $\mathbf{1 7 6 . 0}$ & $\mathbf{7 9 . 9}$ & $\mathbf{2 0 9 . 8}$ \\
\hline m & 2.0 & 2.3 & 2.0 & 30.4 \\
\hline Second group & & & \\
\hline M & $\mathbf{3 7 . 6}$ & $\mathbf{1 7 7 . 0}$ & $\mathbf{7 4 . 0}$ & $\mathbf{9 . 4}$ \\
\hline m & 2.8 & 1.8 & 2.5 & 0.2 \\
\hline
\end{tabular}

\section{Testing procedure}

Muscle function was tested before and after spaceflight in the Exercise Physiology Laboratory at the Johnson Space Center (Houston, Texas, USA) and the Yu.A. Gagarin Cosmonaut Training Center (Star Situ, Moscow Region, Russia).

Torques of extensor and flexor muscles of the back, knee, and ankle were measured during voluntary movements of back, knee, and ankle flexion and extension. All testing (except for the trunk) was unilateral with the dominant (the right) limb, unless otherwise contraindicated (i.e., previous injury). Joint positions (configurations) and ranges of motion were recorded for each subject and reproduced upon testing on day + R5/+ R7 after landing. Peak torques and joint angle at peak torque of various muscles were measured with a LIDO $^{\circledR}$ Active Multi-Joint Isokinetic Rehabilitation System, Loredan Biomedical (USA) [38], which was modified to measure the maximal joint torque during concentric muscle contractions of more than $400 \mathrm{Nm}$. Concentric and eccentric strength were testing in the trunk and upper and lower limbs.

All subjects were instructed to abstain from food for 2 hours before testing, from caffeine for 4 hours before testing, and from exercise for 12 hours before testing.

Before isokinetic testing was conducted, subjects pedaled a cycle ergometer at a workload of 25-50 W at a cadence of 60-80 rpm for $5 \mathrm{~min}$. Standard joint-specific warm-up procedures were followed and consisted of five submaximal repetitions and two to three maximal repetitions. After the warm-up, subjects rested at least $2 \mathrm{~min}$. Strength tests were performed such that subjects exerted a maximal effort in only one direction for each set of repetitions.

A subject sat comfortably on the special universal table of a LIDO $^{\circledR}$ isokinetic dynamometer. The LIDO $^{\circledR}$ dynamometer was calibrated externally and internally (electronically) before each test session. Gravity correction procedure was performed to eliminate the effect of the limb weight on joint torque measurements.

Concentric and eccentric strength were testing in the trunk and lower limbs. Concentric endurance was tested in the knee. Verbal instructions were consistent and given before each joint test. Each subject was instructed to exert maximal effort in only one direction and in every movement when performing a test. No verbal encouragement was given during thew tests.

The subjects familiarized themselves with each angular test velocity by performing three to five movements, from which the maximum value from either of the two pre-flight baseline sessions was selected. Data were collected after 2-3 min rest.

Three protocols were employed in pre- and postflight 
testing for maximal voluntary joint torques produced by various muscles.

In Protocol 1, joint torques were measured during voluntary concentric movements with various angular velocities when flexing and extending back, knee, and ankle muscles.

In Protocol 2, joint torques were measured during voluntary eccentric movements with various angular velocities when flexing and extending back, knee, and ankle muscles.

In Protocol 3, repetitions voluntary concentric movements were assessed upon ankle extension at a velocity of $60^{\circ} / \mathrm{s}^{-1}$.

Each subject performed three movements at a given velocity in a given movement mode with at least $1 \mathrm{~min}$ rest between movements. Prior to isokinetic testing, crewmembers joint-specific warm-up procedures consisted of five submaximal repetitions and two to three maximal repetitions using the actual testing movements and speeds. After the warm-up, crewmemberd rested at least 2-3 min before collction began. Strength tests were performed such that crewmembers exerted a maximal effort in only one direction for each set of repetitions.

Muscle strength was measured as a peak joint torque produced in the total range of concentric (Figure 1, upper panel) or eccentric (Figure 1, middle panel) movements, and the highest value obtained in consecutive voluntary contractions for both flexor and extensor muscles was taken as a maximal joint torque.
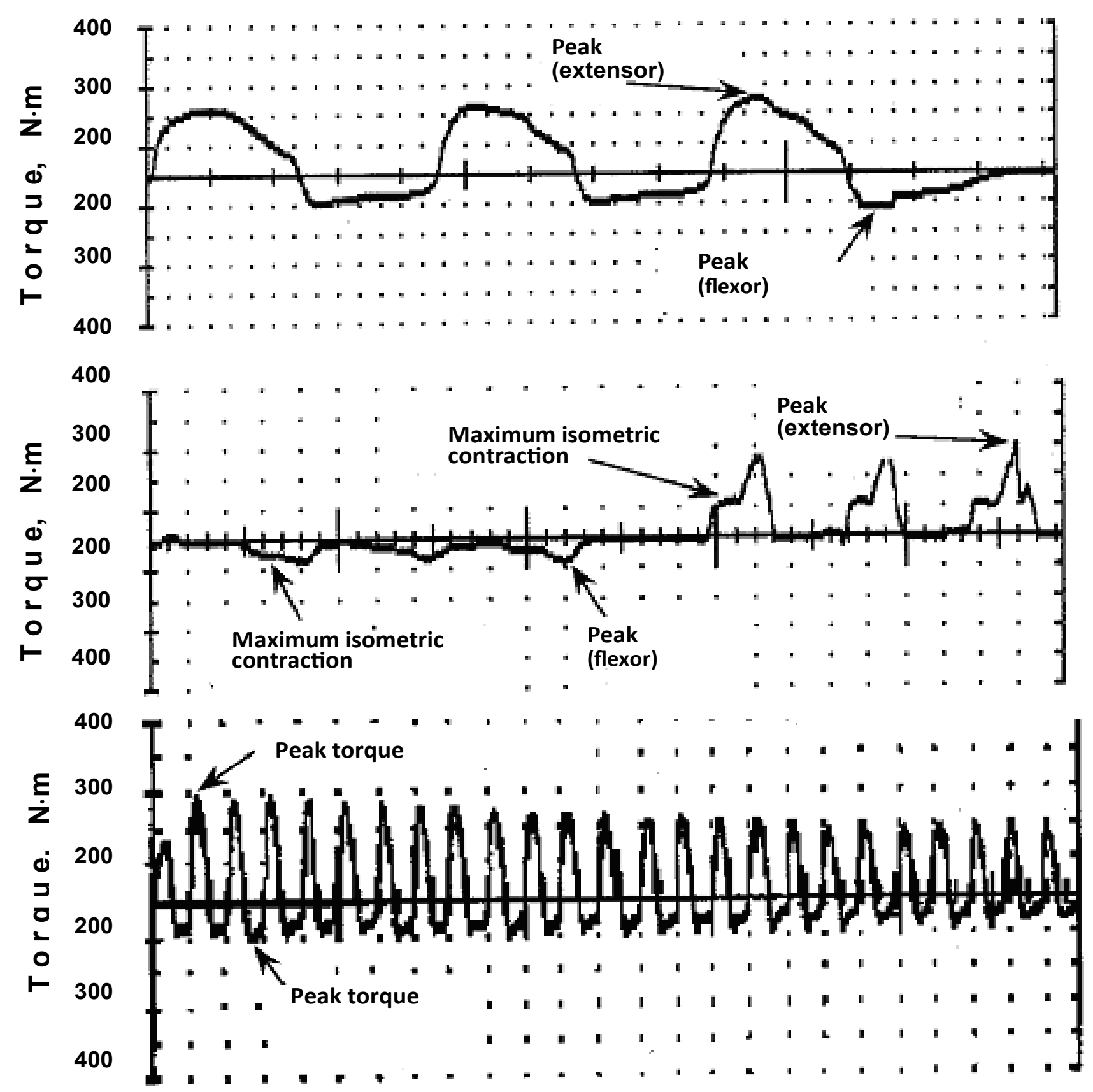

Figure 1: Examples of three concentric (upper panel) and eccentric (middle panel) isokinetic voluntary contractions and during 25 repetitions of isokinetic voluntary contractions (lower panel). 
Testing the flexors and extensors of the back of the subject was carried out in a sitting position and angle of the hip joint was $\sim 130-140^{\circ}$ and hip axis coincides with the axis of rotation of the recording device isokinetic dynamometer (Figure 1, top panel). The range of motion in trunk flexion and extension was $75-130^{\circ}$ at angular velocities of 30 and $75^{\circ} / \mathrm{s}^{-1}$.

Testing the extensor muscles and the hip flexors of the subject was carried out in a sitting position and angle of the knee joint was $\sim 90^{\circ}$, and the axis of the knee joint coincides with the axis of rotation of the recording device of the dynamometer. The range of motion in flexion and extension was $90-60^{\circ}$ in tests for maximal joint torque during concentric movements and work capacity and $33-90^{\circ}$ in tests for maximal joint torque during eccentric movements. Angular velocities of thigh muscle contractions were $30,60,120$, and $180^{\circ} / \mathrm{s}^{-1}$ in concentric movements and $30,60,90$, and $120^{\circ} / \mathrm{s}^{-1}$ in eccentric movements.

Testing muscles, the extensor and flexor of the foot is performed in the test position "kneeling" and the angle of the knee and ankle joint was $\sim 90^{\circ}$ and ankle joint axis coincides with the axis of rotation of the recording device dynamometer (Figure 2). The range of motion in ankle flexion and extension was $\sim 25^{\circ}$ in the case of maximal extension and $20^{\circ}$ in the case of minimal flexion in both concentric and eccentric movements (Figure 1, lower

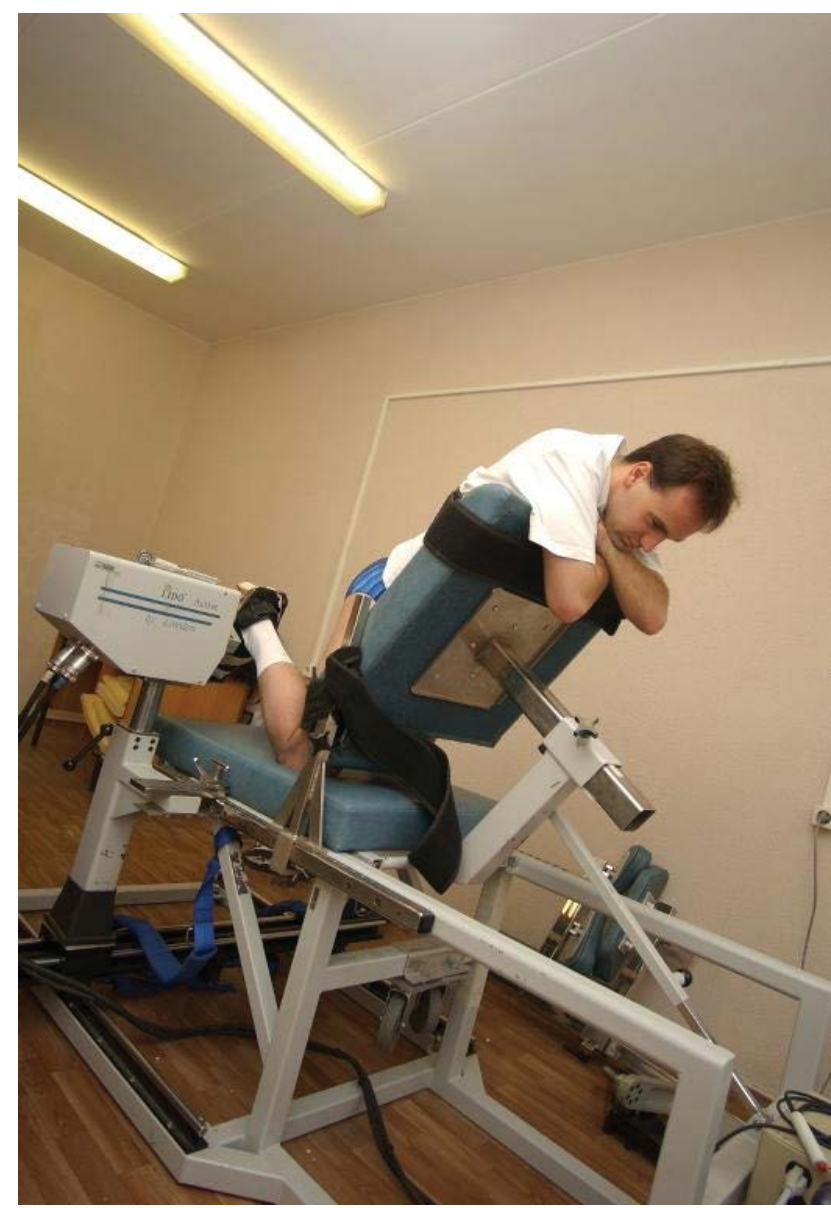

Figure 2: A subject undergoes strength testing with the LIDO $^{\circledR}$ dynamometer (position the subject on his knees). panel). Angular velocities of ankle muscle contractions were 60,120 , and $180^{\circ} / \mathrm{s}^{-1}$ in concentric movements and $30,60,90$, and $120^{\circ} / \mathrm{s}^{-1}$ in eccentric movements.

After voluntary testing, the subjects proceeded with the fatigue test. Skeletal muscle endurance was defined as the total work generated during 25 repetitions of voluntary concentric knee extension at an angular velocity of $60 / \mathrm{s}^{-1}$ in cycles of 1 -s contraction and 1-s relaxation (Figure 1, lower panel). The angle between the thigh and shin was 90 in the starting position. Prior to performing local dosed muscle work, the subject was instructed "to exert maximal effort in every movement".

Peak torque values were used as measures of knee extensor/flexor strengths in pre- and post-spaceflight sessions. For each joint movement, peak torque was defined as the highest torque value achieved across each of the repetitions. For knee extensor and flexor tests, isokinetic endurance was defined as the total work performed during repetitions 2-25. The first prespaceflight rest test was considered a familiarization session and was not used for subsequent analyses.

The degree of fatigue, or performance, was evaluated in terms of Fatigue Index (FI), which was calculated as a ratio of the maximal joint torque averaged over the five last contractions in a series of voluntary 25 repetitions concentric muscle contractions to the maximal joint torque averaged over the five first contractions [39].

\section{Exercise in space}

To preserve the muscle contractile properties in long-term space missions, cosmonauts used PT, which were mostly of a locomotor type and were performed for 1.5-2 hours. Four-day microcycles, each including 3 days with PT and 1 day of rest, were followed in the PT program $[36,37]$.

A treadmill and a cycle ergometer were the main PT tools, training the cardiovascular and respiratory systems $[40,41]$. The treadmill device could be used in a passive (subject driven) or active (motorized) mode of operation, which was selected by the crewmember during each exercise session. Crewmembers used a subject-loading device to fix themselves to the treadmill, which provided varying levels of loading relative to body weight (typical load was $\sim 70 \%$ of body weight) during use. In this way, the crewmembers could complete running or walking exercise while partially loaded. The cycle ergometer provided a load of 50-225 W at a pedaling rate of 40-80 rpm. The crewmembers also had access to bungee cords, which they could use to provide resistance-type exercise for various muscle groups.

\section{Calculations and statistical analysis.}

The effect of spaceflight on function [all isokinetic speeds and Maximal Voluntary Contraction (MVC) angles] was analyzed using a one-way repeatedmeasures ANOVA for each variable. A significant time 
effect was interpreted using pairwise comparisons combined with the Bonferroni adjustment. For all strength measurements, data were collected at $500 \mathrm{~Hz}$. From these data, peak torque, for any given measurement, was taken as the highest recorded value. Any record showing an artifact or torque spike was discarded and not used for analysis. During the maximal isokinetic contraction, the record with the greatest value was used. In addition, the angle-specific torque at $90^{\circ}$ was recorded for all contractions. It should be noted that the muscular results from these experiments reflect those of space travel (which includes a heavy daily work schedule). Differences between preflight (baseline) and postflight parameters were checked for significance by the parametric Student $t$-test and were considered significant at $p<0.05$. Values are given as mean \pm s.e.m. in the text. The percentage changes for pre- and postspaceflight was also calculated.

\section{Results}

\section{Short-term spaceflight}

Our analysis of the functional changes in muscles showed that the maximal joint torques of flexor muscles were consistently lower than baseline in both concentric and eccentric voluntary movements at all angular velocities.

However, the decrease in maximal joint torque of hip extensors substantially varied (Figure 3, upper panel), being the greatest in voluntary concentric movements in a low-velocity high-force mode at angular velocities of 30 and $60 / \mathrm{s}^{-1}$ (by 16 and 13\%, respectively) and the lowest in high-velocity modes at velocities of 120 and $180 \% \mathrm{~s}^{-1}$ (by 9 and $11 \%$, respectively). In the case of hip flexors, the maximal joint torque decreased insignificantly throughout the angular velocity range, by
A
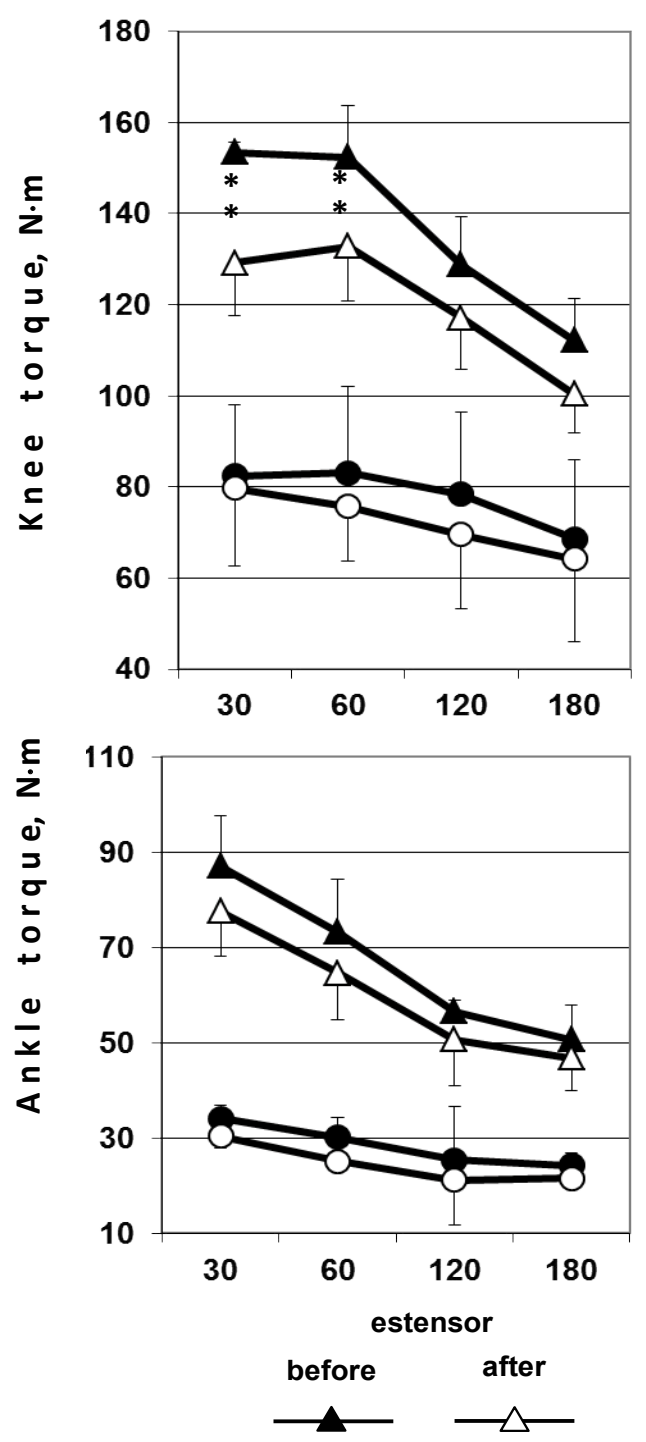

B
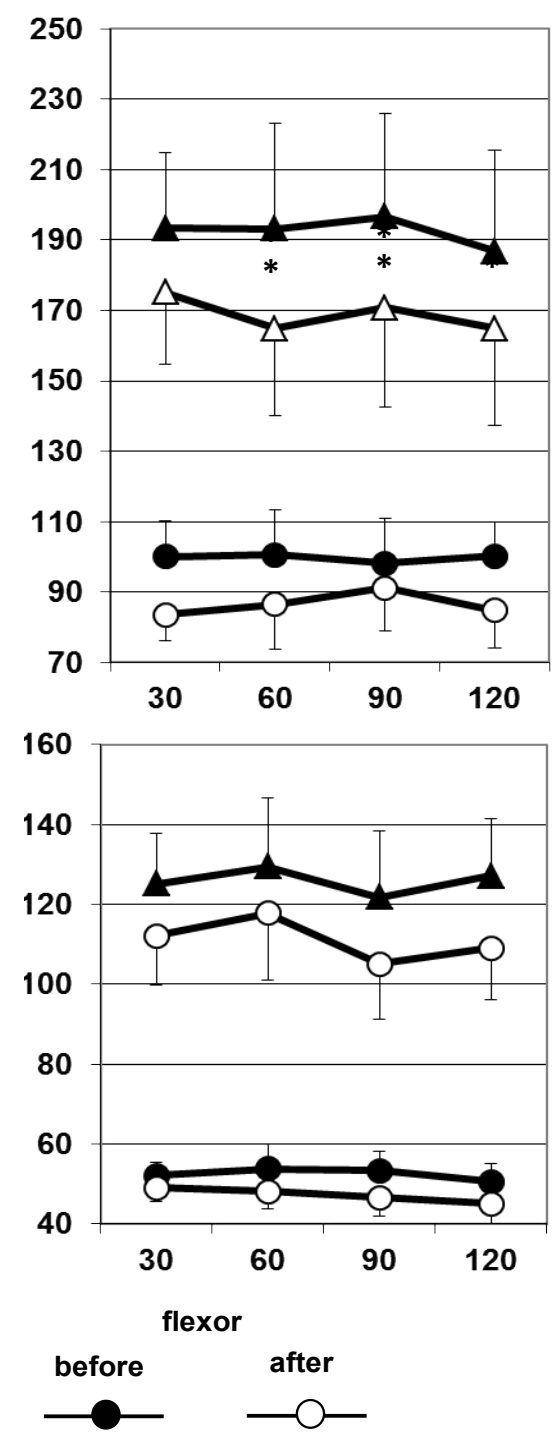

Angular velocity, $\mathrm{deg} / \mathrm{s}^{-1}$

Figure 3: Knee (upper panel) and ankle (lower panel) flexors were tested for maximal joint torque during (a) concentric and (b) eccentric voluntary contractions at various angular velocities. Changes observed after a short-time spaceflight are shown. ${ }^{*} p<0.05 ;{ }^{* *} p<0.01$. 


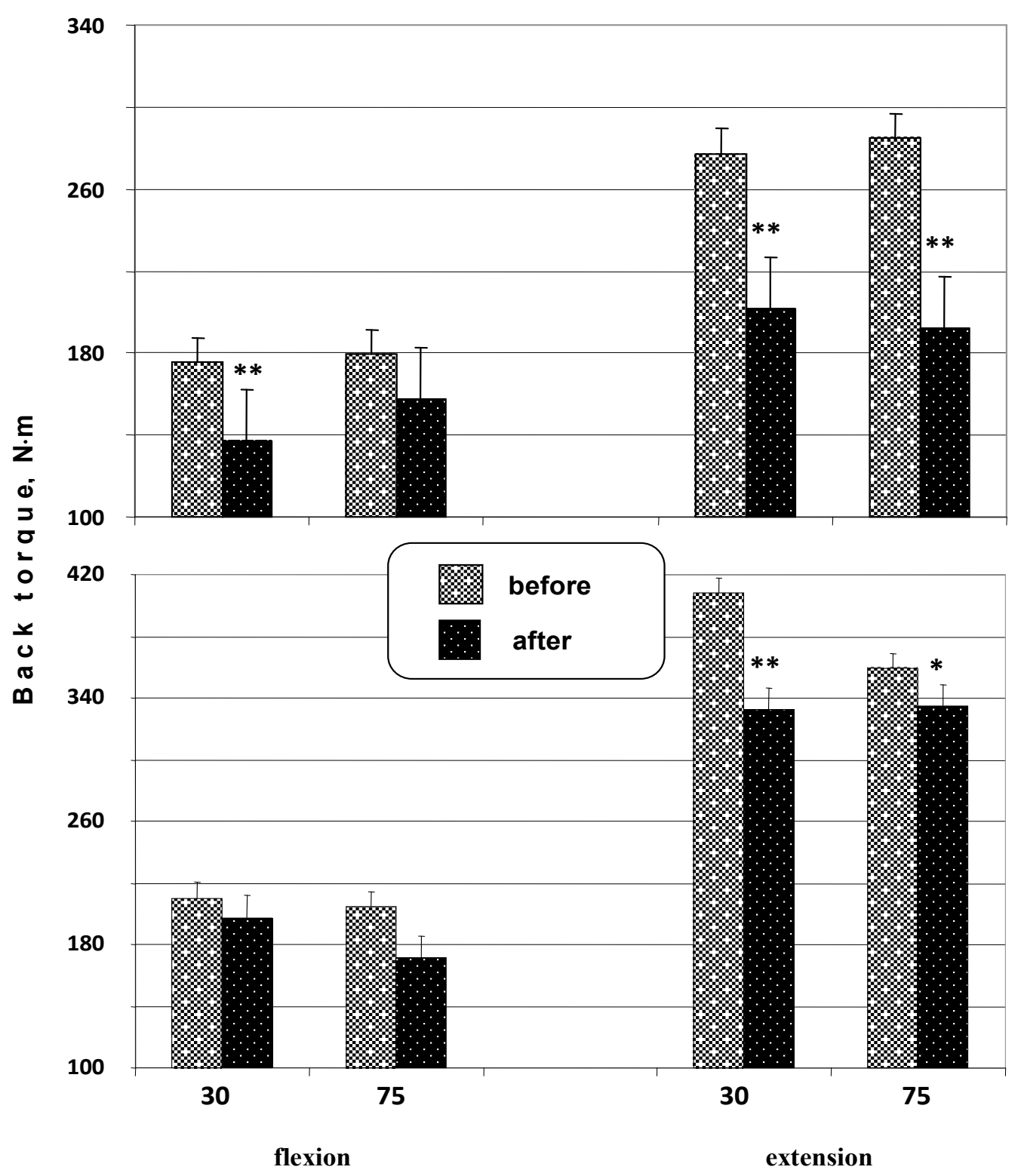

Angular velocity, deg/s $\mathrm{s}^{-1}$

Figure 4: Back flexors and extensors were tested for maximal joint torque during concentric (upper panel) and eccentric (lower panel) voluntary movements at various angular velocities. Changes observed after a short-time spaceflight are shown. ${ }^{*} \mathrm{p}<0.01$.

$3,8,11$, and $6 \%$ at angular velocities of $30,60,120$, and $180^{\circ} / \mathrm{s}^{-1}$, respectively.

When analyzing the maximal joint torques produced by knee muscles during voluntary eccentric isokinetic movements, similar decreases (approximately 14\%) in contractile potential were observed at angular velocities of 60,90 , and $120^{\circ} / \mathrm{s}^{-1}$ and an insignificant increase $(9 \%$ on average) was only detected a low-velocity (highfrequency) regime.

Decreases in maximal joint torque of ankle extensors (Figure 3, lower panel) were much the same (approximately 13\%) in eccentric isokinetic voluntary movements at angular velocities of 30,90 , and $120^{\circ} / \mathrm{s}^{-1}$. In the case of ankle flexors, the lowest changes ( 6\%) were observed for low-velocity (high-force) movements $\left(30^{\circ} / \mathrm{s}^{-1}\right)$.
A greater effect of spaceflights on muscle strength was observed for back muscles in both concentric and eccentric movements at all angular velocities. A decrease in strength of trunk extensors was maximal (33\%) in high-velocity movements and lower (27\%) in low-velocity (high-force) movements (Figure 4). In the case of back flexors, a decrease in maximal joint torque was greater (16\%) at high velocities than at low velocities (high force) (approximately 6\%).

Figure 5 (upper panel) shows how the maximal joint torque of hip extensor muscles changed during rhythmic dynamic concentric movements (contractions) at a constant angular velocity of $60 \% \mathrm{~s}$ before and after a short-term space mission. A data analysis demonstrated that the maximal joint torque of knee extensor muscles generally decreased during rhythmic movements, but 


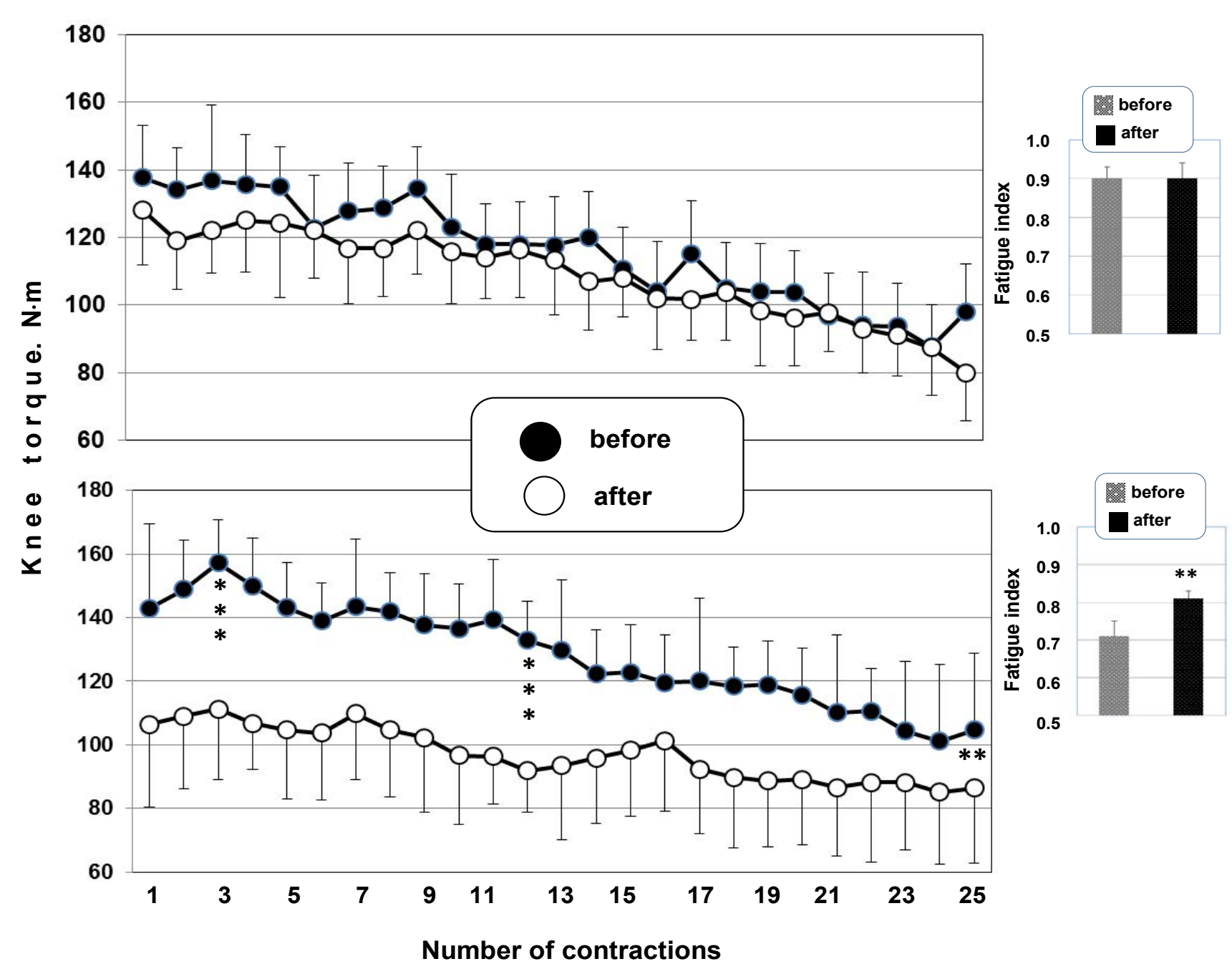

Figure 5: Time course of the maximal joint torque of knee extensors during 25 rhythmic concentric movements (velocity $60 \%$ ) was studied before and after short-term (upper panel) and long-term (lower panel) spaceflight.

${ }^{* *} \mathrm{p}<0.01 ;{ }^{* * *} \mathrm{p}<0.001$.

the kinetics of changes in torque did not significantly differ between pre- and postflight tests; i.e., work capacity of hip extensors, or their fatigue, did not significantly change after a spaceflight.

The $\mathrm{Fl}$ averaged $0.90 \pm 0.03$ at baseline and remained much the same, $0.90 \pm 0.04$, after a spaceflight (Figure 5, upper panel, insert).

\section{Long-duration spaceflight}

An analysis of changes in the force-velocity parameters of knee flexors and extensors (Figure 6, upper panel) showed, first, that the maximal joint torques of the muscles in eccentric movements were substantially higher than in concentric movements. Second, the maximal joint torque of thigh muscles decreased considerably in all crew members at all angular velocities both before and after a spaceflight according to isokinetic dynamometry data.

The extensor muscles showed significant changes in both concentric and eccentric movement modes. The changes differed in extent between the modes, being greater in concentric than in eccentric movements. In the case of hip flexors, similar decreases in force properties (approximately 20\%) were observed at different angular velocities in both concentric and eccentric movements. In the case of hip extensors, such a pattern was not observed, and the changes varied. Substantial changes in maximal joint torque were detected during concentric movements in high-velocity $\left(180^{\circ} / \mathrm{s}^{-1}\right.$; about $25 \%)$ and high-force $(60 \%$ s; about $23 \%)$ modes, while similar decreases in the parameter were seen at angular velocities of $120^{\circ} / \mathrm{s}^{-1}$ and $30^{\circ} / \mathrm{s}^{-1}$.

A similar time course of changes in force-velocity properties was observed for ankle flexors and extensors (Figure 6, lower panel). It should be noted that the maximal joint torques of ankle flexors and extensors changed to a greater extent than those of hip flexors and extensors. Greater changes were observed during movements in both concentric and low-velocity $\left(30^{\circ} / \mathrm{s}^{-1}\right)$ high-force eccentric modes, especially in the latter.

At higher velocities $\left(120^{\circ} / \mathrm{s}^{-1}\right.$ and $\left.180^{\circ} / \mathrm{s}^{-1}\right)$, greater changes were detected in eccentric movements. Ankle flexors showed unidirectional changes, decreasing in maximal joint torque in both concentric and eccentric 
A
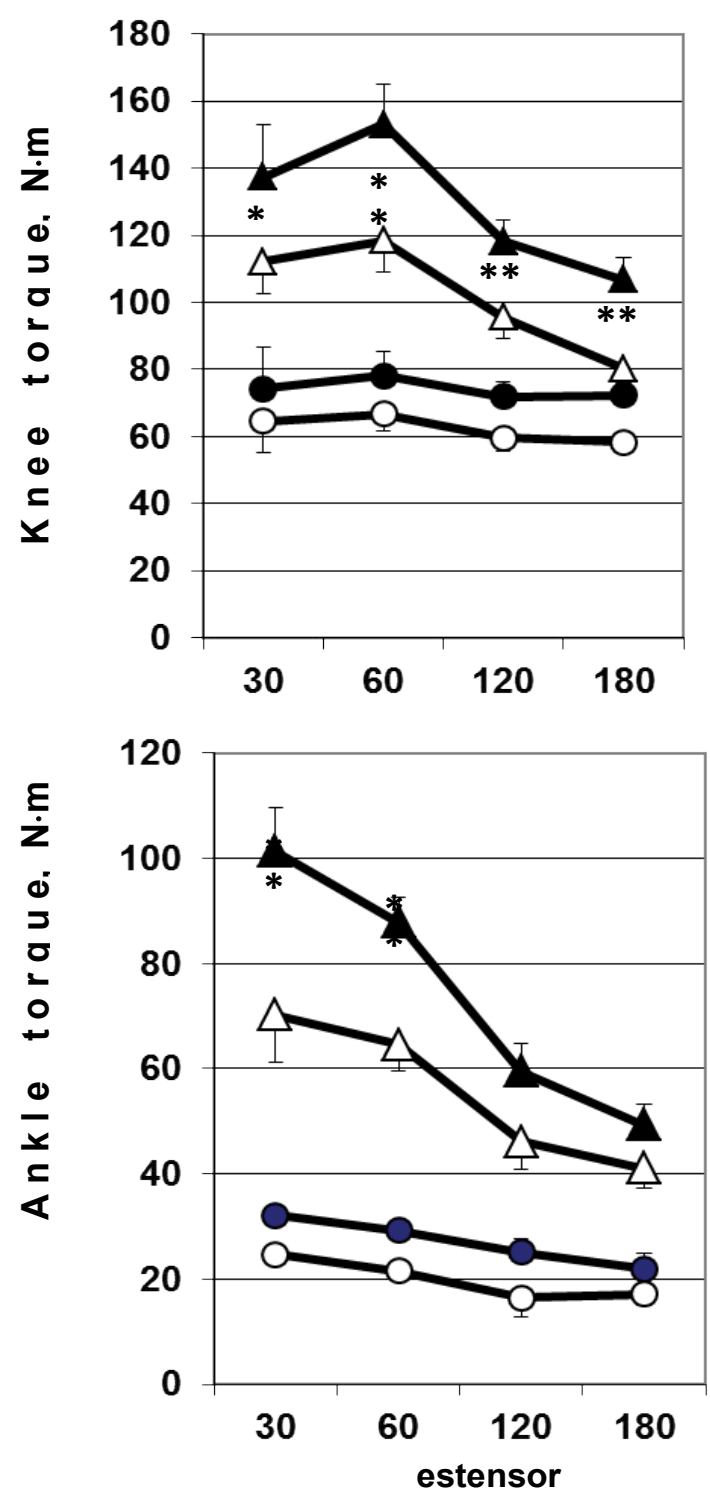

B
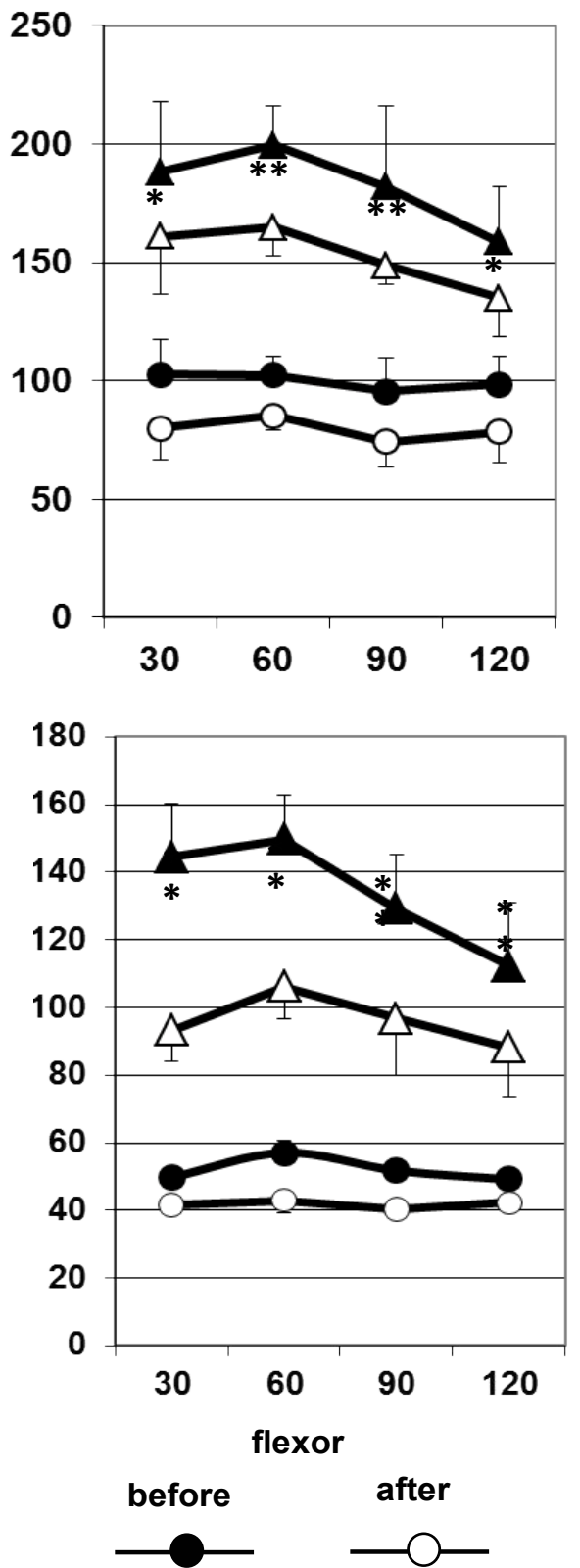

Angular velocity, deg/s ${ }^{-1}$

Figure 6: Knee (upper panel) and ankle (lower panel) extensors (two upper curves) and flexors (two lower curves) were tested for maximal joint torque during concentric (on the left) and eccentric (on the right) voluntary movements at various angular velocities before and after a long-term spaceflight.

${ }^{*} p<0.05 ;{ }^{* *} p<0.01$

movements throughout the angular velocity range examined. The changes in maximal joint torque were somewhat greater in concentric voluntary movements.

An analysis of the changes in maximal joint torques produced by back flexors and extensors during eccentric and concentric movements (Figure 7) showed, first, that the maximal joint torques produced by back flexors and extensors in eccentric contractions were significantly higher than in concentric contractions both before and after a spaceflight. Second, dissimilar muscle responses to unloading were observed during concentric and eccentric voluntary movements. Changes detected during low-velocity concentric contractions of back flexors were significantly lower than during eccentric movements.

Back flexors showed opposite changes. At the same time, concentric voluntary movements at a greater angular velocity $\left(75^{\circ} / \mathrm{s}^{-1}\right)$ revealed an opposite pattern. The greatest changes in flexors were observed during concentric movements compared with eccentric ones, while back extensors displayed the greatest changes during eccentric movements compared with concentric ones.

The time course of changes in the force of knee extensor contractions was analyzed in crew members involved in long-duration space missions (Figure 5, 


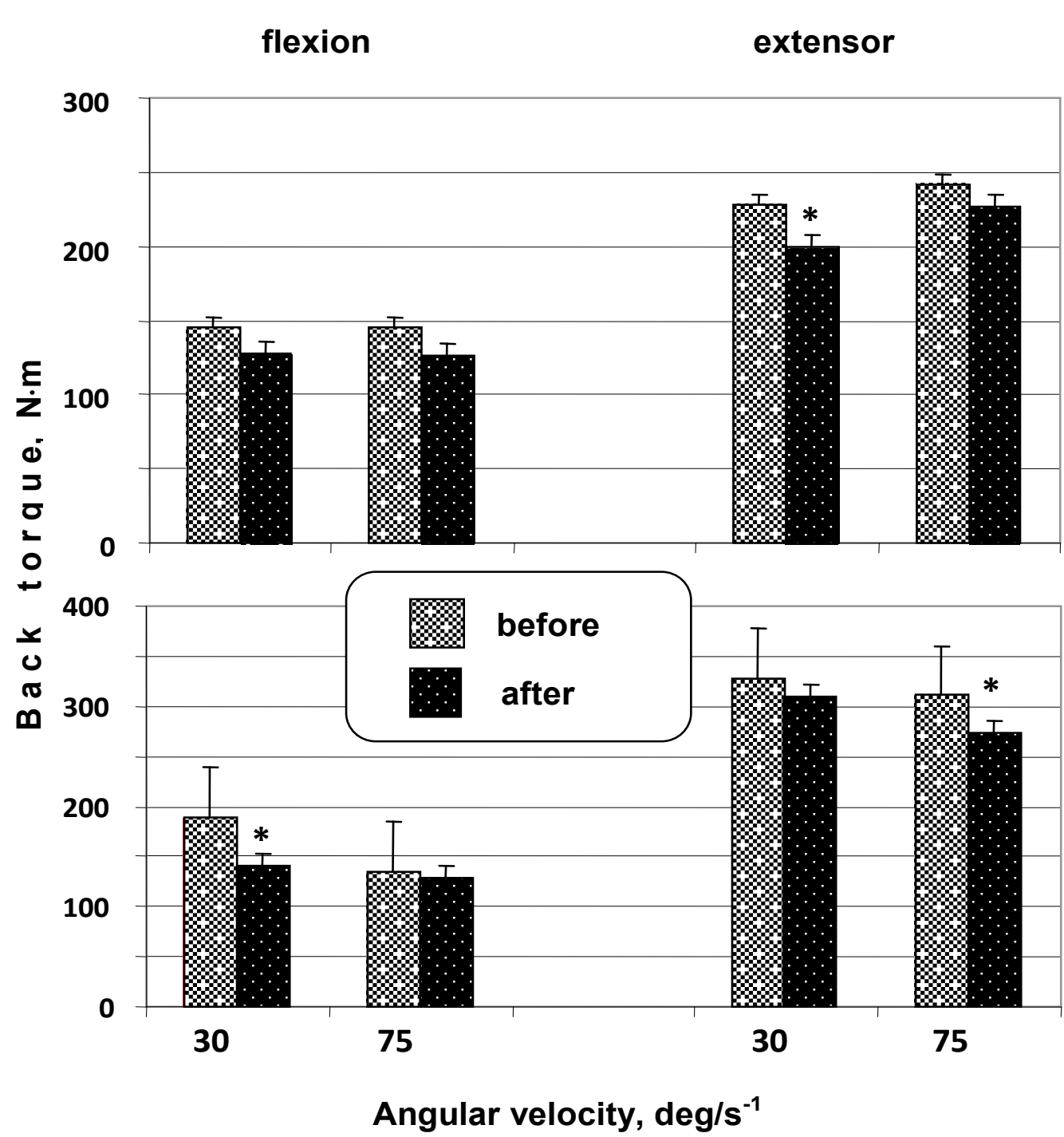

Figure 7: Back extensors and flexors were tested for maximal joint torque during concentric (upper panel) and eccentric (lower panel) voluntary movements at various velocities before and after a long-term spaceflight. ${ }^{*} \mathrm{p}<0.05 ;{ }^{* *} \mathrm{p}<0.01$.

lower panel). Tests for dynamic concentric rhythmic movements (contractions) at a constant angular velocity of $60^{\circ} / \mathrm{s}^{-1}$ were performed before and after a spaceflight. The analysis showed a decrease in contraction force over 25 rhythmic contractions. In other words, the work capacity of knee extensors substantially decreased; i.e., fatigue of the muscular system increased after a spaceflight $(p<0.05)$.

The $\mathrm{Fl}$ averaged $0.71 \pm 0.04$ at the baseline and increased to $0.81 \pm 0.02(p<0.01)$ after a spaceflight (Figure 5, lower panel, insert).

\section{Discussion}

Three primary findings resulted from this study. First, the results of this study contribute to the literature describing the effects of spaceflight on strength various muscle. Second, our results supplement the literature data on the effects of short-term and long-duration spaceflights on the contractile properties of human muscles. A general trend observed in our study is that the maximal joint torques developed by lower-extremity muscles during concentric and eccentric isokinetic contractions decreased both after short-term and longduration space missions. A time course of functional changes arising in various muscle groups was inferred from the experimental findings. Third, we observed that muscles respond to unloading in a nonlinear manner in humans exposed to microgravity, contractile properties decreasing to a greater extent in the flexor muscles that are involved in maintaining vertical posture under normal Earth's gravity (1 G). Changes in extensor muscles were similar, but lower in extent. A direct strength comparison after a long-term spaceflight revealed certain differences for muscle groups involved in locomotion.

As demonstrated in earlier studies focusing on the characteristics of voluntary and electrically induced tetanic contractions of $m$. triceps surae, a short-term spaceflight causes a slight decrease in the forces of MVC (by 9\%) and electrically evoked tetanic contractions (by 8\%) and an increase in force deficiency (by $12 \%$ on average) [42]. Long-duration spaceflights lead to a 
substantial decrease in MVC force (by $22 \%$ ) and, what is of principal importance, muscle intrinsic force properties measured by the force of electrically evoked tetanic contractions (by 16\%), causing almost a twofold increase in force deficiency $[33,43]$. The findings directly indicate that a predominantly central mechanism determines a decrease in muscle contractile properties in a short-time spaceflight (in early exposure to microgravity), while a predominantly peripheral (intramuscular) mechanism comes into play at a next stage. The finding that force deficiency further increases after a long-duration spaceflight indicates that the central mechanisms continue contributing to the decrease in muscle contractile properties.

A decrement in peripheral local muscle work capacity may be due to both a decrease in muscle strength because the total area under the work capacity curve depends on the force of contraction and a poorer ability to maintain (produce) high tension in muscle contraction, that is, a decrease in endurance. The area under the curve decreased substantially for all regions of the contraction curve obtained for a test contraction after a spaceflight. The finding indicates that muscle work capacity was reduced not only because the force decreased, but also because endurance grew lower as well. It is infeasible to determine the specific contribution to work capacity for each particular factor because dynamic voluntary contractions were used to measure the work capacity, and these contractions provide an integral characteristic for both the contractile properties of the muscles involved (a peripheral factor) and their central nervous regulation (a central factor). Changes in the peripheral (contractile) component of the neuromuscular system cannot alone explain the decrease in muscle work capacity, although their contribution is major. A role is also played by alterations of the electrogenic component, including lower propagation velocities of action potentials in motoneuron terminals, neuromuscular synapses [44], and electrogenic membranes of muscle fibers [45-47] As we have shown previously, more prolonged exposure to mechanical unloading of the muscular system leads to a relatively higher contribution of the electrogenic component to peripheral fatigue [44].

Greater changes in contractile properties have been observed by isokinetic dynamometry, wherein many muscles are involved in a given movement, as compared with tendon dynamometry [33,42-44], wherein only one muscle is assessed. The finding suggests activation of antagonist muscles $[5,48,49]$ and a role of efferent input to these muscles $[50,51]$. This input has a relatively low intensity, but seems to increase in microgravity, where support load as a factor that triggers important events in the locomotor system is absent, automatically suppressing (up to complete inhibition?) tonic activity and subsequently causing a number of physiological and structural secondary effects.
Another concept discussed in the spaceflight context is that mechanical unloading change the activation and pattern of recruited motor units, the change affecting the muscle output in movement tests. The simplest conditions for evaluating adaptation of the muscle contractile function are provided by isometric contractions, wherein the muscle length remains constant. However, the muscle length and loading may decrease or increase depending on the external conditions during movements in vivo. In spite of its complexity, the relationship between force and speed at a particular activation level is predictable for many skeletal muscles, given that the muscle architecture and movement mechanics are known. Although the function and work capacity of muscles change in microgravity, basic mechanisms generating muscle force are preserved. The force potential is somewhat higher when an activated muscle elongates at a zero speed. It should also be noted that the force decreases with the increasing contraction speed, and, vice versa, the maximal speed increases when a minimal load is imposed on contractile elements of the muscle.

Our findings show that greater unloading-induced changes in contractile functions occur in extensors compared with other muscles [22,52] after a longduration space mission in spite of regular PT. The finding indicates that PT is not intense enough to preserve the muscle system $[2,53]$.

It follows that the contractile properties of a muscle depend on its activity history in addition to other factors. The cosmonaut training process was therefore analyzed, and the analysis showed that cyclic exercises were mostly employed in training crew members for longterm space missions. Such training seems insufficient for preserving the muscle function during a spaceflight. Hence, the training process should be planned with due regard to the functional potential of the neuromuscular system as a leading physiological system of the body.

The fact that muscle contractile functions decrease during space missions supports the idea that more efficient training programs are needed to protect skeletal muscles in long-term spaceflights. The majority of PT programs used during spaceflights utilize a treadmill and a cycle ergometer as main tools, providing mostly cardiovascular and respiratory training $[40,41,54]$ and improving aerobic capacity, but not power properties of the muscle system. However, the programs have been found to be low efficient not only in maintaining muscle mass and functions, but also in regulating mineral turnover $[55,56]$. As has been observed, the MVC force is already dramatically reduced (40\%) after 7-day unloading without PT and continues to decrease with increasing exposure duration $[4,12,16,44,57-61]$. Changes in neural drive may act as a MVC-limiting factor because muscle contractile properties have been found to decline greater than muscle mass, volume, or CSA in both model 
exposures (bed rest $[28,49]$ ) and spaceflights [6].

Power (resistance) training in $1 \mathrm{G}$ conditions is known to increase muscle strength, muscle size, and neural drive [62]. Power training is therefore a promising means to alleviate muscle disadaptation in microgravity [63]. At any rate, observations have shown that, to maintain the mass and dynamic and isometric forces of hip and ankle extensors, it is enough to do resistance exercises every other day during a 14-day bed rest [6466] or every third day during a 21-day unilateral limb unloading (suspension) [67].

Therefore, the fact that muscle contractile functions decrease, especially in long-term spaceflights, supports the idea that muscle protection during long-duration missions warrants a training program that includes highintensity exercises to improve the power properties of muscles $[2,68,69]$. Power exercises seem promising for preventing muscle atrophy and weakness. Moreover, to make resistance training maximally efficient, a PT program should include both concentric and eccentric muscle contractions performed with a high intensity $[25,62,65]$.

To summarize our results indicate that the reduction of contractile functions in space mission crewmembers supports the idea that "protection" of skeletal muscles during long-term spaceflight requires PT with more effective program. The program of PT with the use of such means of training process as treadmill and bicycle ergometer improves aerobe capacity by providing of training of cardiovascular and respiratory systems [40], but not power characteristics of muscle apparatus. Our findings showed that the program of PT and the exercise devices available on the ISS (the treadmill, and the cycle ergometer, and with the advanced resistive exercise device) not able to elicit loads comparable to exercise on Earth. This is confirmed by other authors [70]. Moreover, the duration and/or loading proved insufficient and to prevent bone loss [71]. That is why the reduction of contractile functions of muscles after spaceflight supports the idea that "protection" of muscles during spaceflight requires PT with more effective training program including in particular highintensive and/or "explosive" exercises which increase power characteristics of muscles $[25,71]$, and are promising for avoiding muscles atrophy and "weakness".

\section{Conclusions}

Our experimental analysis confirmed again that certain phenomena are seen after short-term and long-duration spaceflights, that is, exposure to real microgravity. We quantitatively described the postflight changes and assumed that at least two mechanisms are responsible for the decrease in muscle contractile functions, determining its two stages. A central mechanism mostly contributes to the decrease in contractile functions in early exposure to microgravity (short-time spaceflights), while a peripheral mechanism comes to play a major role at the second stage (longduration spaceflights).

\section{Acknowledgments}

The author appreciate wonderful collaboration and is grateful to the NASA's Exercise Physiology Laboratory for providing the isokinetic dynamometer LIDO $^{\circledR}$ and the isokinetic data collection. The author am grateful to the "MIR" and ISS crew members who agreed to take part in the study, which would be infeasible without their efforts; medical and engineering staff of the Exercise Physiology Laboratory at the Johnson Space Center (Houston, Texas, USA) and of Yu.A. Gagarin Cosmonauts Training Center (Star City, Moscow Region, Russia), for their contribution to the organization of the study and numerous unnamed specialists who helped to perform the study. The experiments comply with the current laws of the country in which they were performed.

The author also thanks the scientific researchers Steven Siconolfi, Ph.D., and John Gilbert III, Ph.D., who contributed to the gravitational physiology.

The study is supported by Ministry of Education and Science of the Russian Federation (RFMEFI61317X0074) and the author would like to thank Ministry of Education and Science of the Russian Federation for funding this research project.

\section{Conflict of Interest}

There are no conflicts of interest.

\section{References}

1. LeBlanc A, Lin C, Shackelford L, Sinitsyn V, Evans $H$, et al. (2000) Muscle volume, MRI relaxation times (T2), and body composition after spaceflight. J Appl Physiol (1985) 89: 2158-2164.

2. Tesch PA, Thorsson A, Colliander EB (1990) Effects of eccentric and concentric resistance training on skeletal muscle substrates, enzyme activities and capillary supply. Acta Physiol Scand 140: 575-580.

3. Akima H, Kawakami $\mathrm{Y}$, Kubo K, Sekiguchi $\mathrm{C}$, Ohshima $\mathrm{H}$, et al. (2000) Effect of short-duration spaceflight on thigh and leg muscle volume. Med Sci Sports Exerc 32: 1743-1747.

4. LeBlanc A, Rowe R, Schneider V, Evans H, Hedrick T (1995) Regional muscle loss after short duration spaceflight. Aviat Space Environ Med 66: 1151-1154.

5. Edgerton VR, Zhou MY, Ohira $Y$, Klitgaard H, Jiang B, et al. (1995) Human fiber size and enzymatic properties after 5 and 11 days of spaceflight. J Appl Physiol (1985) 78: 17331739.

6. Fitts RH, Riley DR, Widrick JJ (2001) Functional and structural adaptations of skeletal muscle to microgravity. J Exp Biol 204: 3201-3208.

7. Narici MV, Kayser B, Barattini P, Cerretelli P (1997) Changes in electrically evoked skeletal muscle contractions during 17-day spaceflight and bed rest. Int J Sports Med 18: 290-292.

8. Zange J, Müller K, Schuber M,Wackerhage H, Hoffmann U, 
et al. (1997) Changes in calf muscle performance, energy metabolism, and muscle volume caused by long-term stay on space station MIR. Int J Sports Med 18: 308-309.

9. Genin AM, Sorokin PA (1969) Long-term mobility restriction as a model of the effect of microgravity on the human body. In: Long-Term Mobility Restriction and Its Effect on the Human Body. Moscow: Nauka, 9-16.

10. Alkner BA, Tesch PA (2004) Knee extensor and plantar flexor muscle size and function following 90 days of bed rest with or without resistance exercise. Eur J Appl Physiol 93: 294-305.

11. Antonutto G, Capelli C, Girardis M, Zamparo P, di Prampero PE (1999) Effects of microgravity on maximal power of lower limbs during very short efforts in humans. J Appl Physiol 86: 85-92.

12. Berg HE, Larsson L, Tesch PA (1997) Lower limb skeletal muscle function after 6 wk of bed rest. J Appl Physiol (1985) 82: 182-188.

13. de Boer MD, Maganaris CN, Seynnes OR, Rennie MJ, Narici MV (2007) Time course of muscular, neural and tendinous adaptations to 23 day unilateral lower-limb suspension in young men. J Physiol 583: 1079-1091.

14. Kawakami Y, Akima H, Kubo K, Muraoka Y, Hasegawa $\mathrm{H}$, et al. (2001) Changes in muscle size, architecture, and neural activation after 20 days of bed rest with and without resistance exercise. Eur J Appl Physiol 84: 7-12.

15. LeBlanc AD, Schneider VS, Evans HJ, Pientok C, Rowe R, et al. (1992) Regional changes in muscle mass following 17 weeks of bed rest. J Appl Physiol (1985) 73: 2172-2178.

16. Kakurin LI, Cherepakhin MA, Pervushin VI (1971) Effect of short-term spaceflights on the state of the human neuromuscular system. Kosm Biol Med 5: 53-56.

17. Berg HE, Tesch PA (1996) Changes in muscle function in response to 10 days of lower limb unloading in humans. Acta Physiol Scand 157: 63-70.

18. Greenisen M, Hayes I, Siconolfi S, Moore A (1999) Functional performance evaluation in: Extended Duration Orbiter Medical Project Final Report 1989-1995. In: Sawin CF, Taylor GR, Smith WL, NASA Johnson Space Cellter. Houston, TX, NASA SP-1999-534, 3.1-3.24.

19. Tesch PA, Berg HE, Bring D, Evans HJ, LeBlanc AD (2005) Effects of 17-day spaceflight on knee extensor muscle function and size. Eur J Appl Physiol 93: 463-468.

20. Grigor'eva LS, Kozlovskaya IB (1987) Effect of microgravity and hypokinesia on the force-velocity properties of human muscles. Kosm Biol Aviakosm Med 21: 27-30.

21. Kozlovskaya IB, Aslanova IF, Grigorieva LS, Kreidich YuV (1982) Experimental analysis of motor effects of weightlessness. Physiologist 25: S49-S52.

22. Kozlovskaya IB, Grigor'eva LS, Gevlich GI (1984) Comparative analysis of the effects on force-velocity properties and tone of human skeletal muscles for microgravity and its models. Kosm Biol Aviakosm Med 18: 22-26.

23. Lambertz D, Pérot C, Kaspranski R, Goubel F (2001) Effects of long-term spaceflight on mechanical properties of muscles in humans. J Appl Physiol (1985) 90: 179-188.

24. Lee SMC, Cobb K, Loehr JA, Nguyen D, Schneider SM (2004) Footground reaction force during resistive exercise in parabolic flight. Aviat Space Environ Med 75: 405-412.

25. Trappe S, Costill D, Gallagher P, Costill D, Gallagher $P$
(2009) Exercise in space: human skeletal muscle after 6 months aboard the International Space Station. J Appl Physiol 106: 1159-1168.

26. Convertino VA (1990) Physiological adaptations to weightlessness: effects on exercise and work performance. Exerc Sport Sci Rev 18: 119-166.

27. Adams GR, Caiozzo VJ, Baldwin KM (2003) Skeletal muscle unweighting: spaceflight and ground-based models. J Appl Physiol (1985) 95: 2185-2201.

28. Koryak YuA, Kozlovskaya IB (1994) Excitability of the human motoneuron pool in long-term antiorthostatic hypokinesia (HDT). Proc VII All-Russia Conf "Ecological and Physiol Problems of Adaptation", 127-128.

29. Kozlovskaya IB, Burlachkova NI (1997) Effects of microgravity on spinal reflex mechanisms. $12^{\text {th }}$ Man in Space Symp, 310-311.

30. Hather BM, Adams GR, Tesch PA, Dudley GA (1992) Skeletal muscle responses to lower limb suspension in humans. J Appl Physiol (1985) 72: 1493-1498.

31. Koryak YuA (2016) Influence of long-duration space flight on human skeletal muscle architecture and function. A pilot study. Amer Sci J No 6: 7-13.

32. Duchateau J (1995) Bed rest induces neural and contractile adaptations in triceps surae. Med Sci Sports Exerc 27: 1581-1589.

33. Koryak YuA (2001) Neuromuscular adaptation to short- and long-term spaceflights. ISS. Kosm Biol Med. In: Grigor'eb AI, Uschakov IB, Biomedical research on the Russian segment of the ISS, Institute of Biomedical Prob, Russian Academy of Sci. Russian Segment 2: 93-123.

34. Ruegg DG, Kakebeeke TH, Gabriel JP, Bennefeld M (2003) Conduction velocity of nerve and muscle fiber action potentials after a space mission or a bed rest. Clin Neurophysiol 114: 86-93.

35. Oganov VS, Gurfinkel' VS, Kozlova IG, Rachmanov AS, Magedov VS (1991) Effects of long-term hypokinesia and microgravity on the functional state of human skeletal muscles: Experience of using electromechanical efficiency as a criterion. Fiziol Chel 17: 35-47.

36. Eremin Al, Bazhanov VV, Marishchuk VL, Stepantsov VI, Dzhamgarov TT (1969) Human training in conditions of long-term hypokinesia. Prob of Space Biol Moscow: Nauka, 191-199.

37. Stepantsov VI, Tikhonov MA, Eremin AV (1972) Physical training as a method of preventing the hypodynamic syndrome. Kosm Biol Med 6: 64-68.

38. LIDO ${ }^{\circledR}$ (1988) Active Operations Manual.

39. Koryak YuA, Polyakov VV, Potsepaev AI, Mart'yanov VA (1975) Study of the dynamic work capacity of the peripheral neuromuscular system in athletes. Proc I All-Union Conf "Physiological Basis of Movement Control", 73-74.

40. Grigoriev AI, Ditlajn LF, Kozlovskaya IB, Coyen CF (2001) Preventive measures in the short- and long-term space flights. Health Performance Safety of Space Flight. In: Dietlein LF, Pestov ID, Amer Institute of Aeronautics and Astronautics. Washington DC - Sci M, 4: 252-309.

41. Kozlovskaya IB, Grigoriev Al (2004) Russian system of countermeasures on board of the International Space Station (ISS): the first results. Acta Astronaut 55: 233-237.

42. Koryak YuA, Gidzenko Yu, Shattlufort ME, Zaletin S, Lonchakov $\mathrm{Yu}$ (2007) Functional properties of the 
neuromuscular system and their changes after a seven-day mission to the International Space Station. Successes of Modern Natural Sci 12: 149-150.

43. Koryak YA (2015) Influence of 120-Day Stimulated Microgravity with Countermeasures on Human Muscle Musculo-Tendinous Stiffness and Contractile Properties. Int J Sports Exerc Med 1: 1-8.

44. Koryak $Y$ (1998) Electromyographic study of the contractile and electrical properties of the human triceps surae muscle in a simulated microgravity environment. J Physiol 510: 287-295.

45. Bezanlilla F, Caputo C, Gonzalez-Serratos H, Venosa RS (1972) Sodium dependence of the inward spread of activation in isolated twitch muscle fibers of the frog. $J$ Physiol 223: 507-523.

46. Bigland-Ritchie BR, Jones DA, Woods JJ (1979) Excitation frequency and muscle fatigue: electrical responses during human voluntary and stimulated contraction. Exp Neurol 64: 414-427.

47. krnjevic K, Miledi R (1958) Failure of neuromuscular propagation in rats. J Physiol 140: 440-461.

48. Mart'yanov VA (1974) Extent to which the force-velocity potential of the neuromuscular system is utilized in voluntary exertion. Fiziol Zh USSR 60: 1416-1422.

49. Marsden CD, Odeso JA, Rothwell JC (1982) Action of the antagonist muscle during fast limb movements in man. $J$ Physiol 312: 47-48.

50. Mart'ianov VA, Koriak luA (1973) [Increase in voluntary strength under the action of additionally evoked afferent influences]. Fiziol Zh SSSR Im I M Sechenova 59: 17561760.

51. Mart'yanov VA, Kopylov YuA, Gnutov MI (1972) Extent to which the potential of the muscle system is utilized in maximal voluntary exertion. Fiziol Zh USSR 58: 1390-1396.

52. Akima H, Kubo K, Imai M, Kubo K, Imai M, et al. (2001) Inactivity and muscle: effect of resistance training during bed rest on muscle size in the lower limb. Acta Physiol Scand 172: 269-278.

53. Lee SMC, Guilliams ME, Siconolfi SF, Greenisen MC, Schneider SM, et al. (2000) Concentric strength and endurance after long duration spaceflight. Official of $\mathrm{J}$ the Amer College of Sports Med 32: 363.

54. Hargens AR (1994) Recent bed rest results and countermeasure development at NASA. Acta Physiol Scand Suppl 616: 103-114.

55. Morukov BV, Larina IM, Grigor'ev Al (1998) Changes in calcium metabolism and its regulation in humans during long-duration space flight. Human Physiology 24: 102-107.

56. Oganov VS, Bogomolov VV (2009) Human bone system in microgravity: Study review, hypotheses, and possibility to predict the state in long-term interplanetary expeditions.
Aviakosm Ekol Med 1: 3-12

57. Gogia P, Schneider VS, LeBlanc AD, Krebs J, Kasson C, et al. (1988) Bed rest effect on extremity muscle torque in healthy men. Arch Phys Med Rehabil 69: 1030-1032.

58. Koryak YuA (1998) Influence of 120-days $6^{\circ}$ head-down tilt bed rest on the functional properties of the neuromuscular system in man. Aviat Space Environ Med 69: 776-770.

59. Koryak YuA (2010) Functional properties of the human neuromuscular system and their changes as a result of mechanical unloading for various periods of time. FiziolZh 57: 76-89.

60. Koryak Yu (1998) The effect of 120-days of bed rest with and without countermeasures on the mechanical properties of the triceps surae muscle in young female. Eur J Appl Physiol 78: 128-135.

61. Koryak YuA (2011) Adaptation of Human Skeletal Muscle to the Load Change. Experimental study. Germany: LAP LAMBERT Academic Publishing $\mathrm{GmbH}$.

62. Sale DG (1988) Neural adaptation to resistance training. Med Sci Sports Exerc 20: 135-145.

63. Tesch PA, Berg HE (1997) Resistance training in space. Int J Sports Med 18: S322-S324.

64. Baldwin KM, White TP, Arnaud SB, Edgerton VR, Kraemer WJ, et al. (1996) Musculoskeletal adaptations to weightlessness and development of effective countermeasures. Med Sci Sports Exerc 28: 1247-1253.

65. Bamman MM, Clarke MS, Feeback DL, Talmadge RJ, Stevens BR, et al. (1998) Impact of resistance exercise during bed rest on skeletal muscle sarcopenia and myosin isoform distribution. J Appl Physiol 84: 157-163.

66. Bamman MM, Hunrler GR, Stevens BR, Hunter GR, Stevens BR, et al. (1997) Resistance exercise prevents plantar flexor deconditioning during bed rest. Med Sci Sports Exerc 29: 1462-1468.

67. Schulze K, Gallagher P, Trappe S (2002) Resistance training preserves skeletal muscle function during unloading in humans. Med Sci Sports Exerc 34: 303-313.

68. Häkkinen K (1994) Neuromuscular adaptation during strength training, aging, detraining, and immobilization. Clin Rev Phys Rehabil Med 6: 161-198.

69. Tesch PA (1991) Training for bodybuilding. In: The encyclopedia of sports medicine: "Strength and Power in Sports". In: Komi PA, Blackwell Scientific Publications, Oxford, 370-380.

70. Genc KO, Gopalakrishnan R, Kuklis MM, Maender CC, Rice AJ, et al. (2010) Foot forces during exercise on the International Space Station. J Biomech 43: 3020-3027.

71. Cavanagh PR, Genc KO, Gopalakrishnan R, Kuklis MM, Maender CC, et al. (2010) Foot forces during typical days on the international space station. J Biomech 43: 2182-2188. 